\section{Intriguing Localisation of "Pacinian Neuroma" Diagnosed at Intraoperative Consultation during Whipple Procedure}

Sir,

Pacinian corpuscles are receptors that are responsible for pressureand vibration detection. ${ }^{1}$ They are commonly seen in subcutaneous fatty tissue of hand, feet and dorsal aspects of fingers. Less frequently, they are located in conjunctiva, joints and ligaments, urethra, penis, vulva, mesentery, parietal peritoneum and pancreas. ${ }^{1-4}$ Pacinian neuroma is benign proliferation of normal or enlarged pacinian corpuscles.

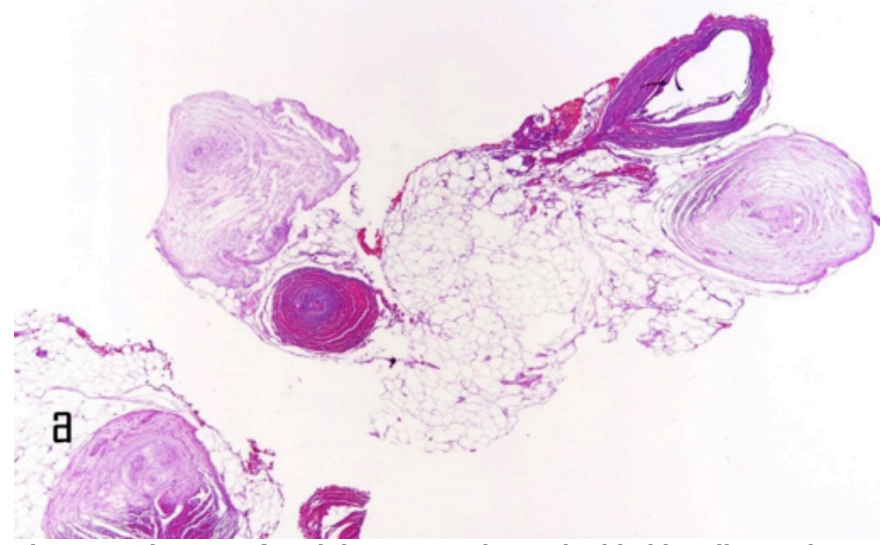

Figure 1: Clusters of pacinian corpuscles embedded in adipose tissue. (H\&E, ×20).

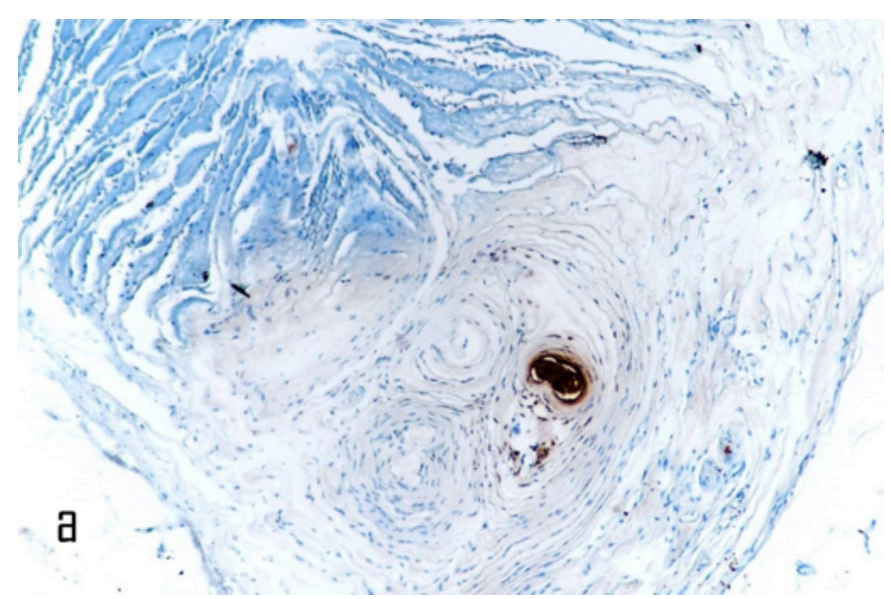

Figure 2: S-100 positivity in terminal nerve. $(\times 100)$.

A 70-year male patient presented to the clinic with complaints of cholelithiasis. During investigation, contrast-enhanced abdominal magnetic resonance imaging showed stones with the largest measuring $14 \mathrm{~mm}$ in the gallbladder, hepatostea- tosis, and a splenic cystic mass. In addition to these findings, a $3.5 \times 2.5 \mathrm{~cm}$ mass lesion with a cystic component was detected in the pancreatic body. With these findings, a decision was made for surgery. During operation, tissue samples with initial diagnosis of metastatic lymph node or tumor implant were sent to the laboratory. Intraoperative examination of the material showed clusters of Pacinian corpuscles in the fatty tissue. There was no any lymphoid tissue or tumor implant. Paraffin sections showed similar histopathologic features (Figure 1). Immunohistochemically, terminal nerve, and onion skin-like cell layers around the terminal nerve were stained with S-100 and EMA (Figure 2). With these findings, final diagnosis of 'Pacinian neuroma' was established. In the macroscopic examination of subtotal pancreatectomy specimen, an irregular, hard, dirty white colour $2.3 \times 1.9 \times 1.2 \mathrm{~cm}$ tumor with cystic areas, with the largest measuring $1 \mathrm{~cm}$ were observed. In the dissection of peripancreatic fatty tissue, six lymph nodes were revealed. In the macroscopic examination of gallbladder and spleen, black colour gallstones and $1 \mathrm{~cm}$ cystic mass were observed. Microscopically, pancreatic tumor was diagnosed as ductal adenocarcinoma. In addition, chronic cholecystitis, cholelithiasis, splenic pseudocyst, and reactive lymphoid hyperplasia were observed in histopathologic examination.

Although the exact pathogenesis of Pacinian neuroma is not clear, repeated traumas are reported in the literature. ${ }^{2}$

Cases diagnosed during surgical operation due to other indications, similar to this case, are very rare. ${ }^{3,4}$

Although, the lesion may resemble fatty tissue macroscopically, microscopic features are characteristic. Histopathologically, it may be composed of either one hypertrophic Pacinian corpuscle or numerous corpuscles. ${ }^{1}$

Although, nerve sheath myxoma is considered in the differential diagnosis, it can be excluded by morphological and immunohistochemical findings. ${ }^{1}$

In conclusion, Pacinian neuroma is a rare entity and its extraordinary localisation, as in this case, should be kept in mind in daily routine and frozen section consultations.

\section{CONFLICT OF INTEREST:}

The authors declared no conflict of interest.

\section{AUTHORS' CONTRIBUTION:}

SSE, GK, MO: Conception of the work, acquisition, analyses or interpretation of data for the work, drafting the work or revising it critically for important intellectual content. Final approval of the version to be published.

\section{REFERENCES}

1. Miettinen M. Nerve sheath tumors. In: Miettinen M, editor. Modern soft tissue pathology, tumors and non-neoplastic conditions. New York Cambridge University Press 2010; p.660-723.

2. Fassola I, Wenzke L, Ertel W, Krasnici S. Pacinian neuromas 
and neurofibromas of the hands and fingers: A systematic review. J Hand Surg Eur 2019; 44(9):925-31. doi: 10.1177/ 1753193419852118.

3. Dembinski AS, Jones JW. Intra-abdominal pacinian neuroma: A rare lesion in an unusual location. Histopathology 1991; 19(1):89-90. doi: 10.1111/j.1365-2559.1991.tb00899.x.

4. Zappacosta R, Zappacosta B, Di Giovannantonio L, Castrataro A, Bellocci R, Angelucci D. Retroperitoneal Pacinian neuroma: Report of a rare lesion in an uncommon location. Pathologica 2004; 96(2):49-51.

Selma Sengiz Erhan', Gamze Kulduk ${ }^{2}$ and Mehmet Ozer
${ }^{1}$ Department of Pathology, Prof. Dr. Cemil Tascıoglu City Hospital, Istanbul, Turkey

${ }^{2}$ Department of Pathology, Bursa Yuksek Ihtisas Education and Research Hospital, Bursa, Turkey

Correspondence to: Dr. Selma Sengiz Erhan, Department of Pathology, Prof. Dr. Cemil Tascioglu City Hospital, Istanbul, Turkey

E-mail: selmaserhan@hotmail.com

Received: November 16, 2020; Revised: February 09, 2021; Accepted: April 23, 2021

DOI: https://doi.org/10.29271/jcpsp.2021.12.1520 